\title{
Urinary tract dilation illustrations: reply to Phelps et al.
}

\author{
Hyun Gi Kim ${ }^{1}$ (D)
}

Received: 30 May 2017 / Accepted: 6 June 2017 / Published online: 2 August 2017

(C) Springer-Verlag GmbH Germany 2017

\section{Dear Editors,}

First of all, I would like to thank Dr. Phelps and his colleagues [1] for their comments on the article, "Conversion and reliability of two urological grading systems in infants: the Society for Fetal Urology and the urinary tract dilatation classifications system" [2].

His point regarding the differences between our illustration and the new illustration, which was vetted by the creators of the urinary tract dilation (UTD) classification system, is well understood. Our illustration was based on the original ultrasound images by Nguyen et al. [3]. We interpreted Figs. 7a and $b$ of their paper, which were sample images of UTD P1, as showing some fluid outlining the medullary pyramid. However, it is now obvious that these images should not have been interpreted as such, and we expect that this kind of misinterpretation will be eliminated with your new illustration.

One other thing I would like to comment on is the usage of the term "dilation" for calyces in the UTD classification system. If the presence of fluid at the central or peripheral calyces is the only matter of concern when grading, I cautiously suggest using the term "visualization" instead of "dilation" to further decrease any possible confusion among users of the UTD classification system. This is because we often use "dilation" when there is blunting of calyces. The term "visualization" of calyces was originally used in the Society for Fetal
Urology grading system [4], so its usage would not be an arbitrary decision.

Once again, I appreciate your efforts to make the new classification system widely known to both radiologists and clinicians and to make it clearly comprehensible and adaptable to clinical practice.

\section{Compliance with ethical standards}

Conflicts of interest None

\section{References}

1. Phelps AS, Chow JS, Back SJ et al (2017) Urinary tract dilation illustrations. Pediatr Radiol 47. doi:10.1007/s00247-017-3916-8

2. Han M, Kim HG, Lee JD et al (2017) Conversion and reliability of two urological grading systems in infants: the Society for Fetal Urology and the urinary tract dilatation classifications system. Pediatr Radiol 47:65-73

3. Nguyen HT, Benson CB, Bromley B et al (2014) Multidisciplinary consensus on the classification of prenatal and postnatal urinary tract dilation (UTD classification system). J Pediatr Urol 10:982-998

4. Fernbach S, Maizels M, Conway J (1993) Ultrasound grading of hydronephrosis: introduction to the system used by the Society for Fetal Urology. Pediatr Radiol 23:478-480
Hyun Gi Kim

catharina315@aumc.ac.kr

1 Department of Radiology,

Ajou University School of Medicine,

Ajou University Medical Center,

164 World cup-ro, Yoengtong-gu, Suwon 443-380, Korea 